\title{
Characterization of the SMYD Family of Lysine Methyltransferases: Reflections upon Key Findings and Therapeutic Implications
}

\author{
Melissa A. Edwards ${ }^{1}$, Jenna Hoobler², Mark A. Brown ${ }^{3,4}$
}

\author{
${ }^{1}$ Cell and Molecular Biology Program, Colorado State University, Fort Collins, CO \\ ${ }^{2}$ Department of Biomedical Sciences, Colorado State University, Fort Collins, CO \\ ${ }^{3}$ Department of Clinical Sciences, Colorado State University, Fort Collins, CO \\ ${ }^{4}$ Epidemiology Section, Colorado School of Public Health, Fort Collins, CO
}

\begin{abstract}
It has been almost two decades since studies upon the SET-MYND (SMYD) family of lysine methyltransferases were first initiated following the serendipitous discovery of the SMYD1 gene. In that time, much has been learned about the roles of the SMYD family in processes ranging from embryogenesis to tumorigenesis. Herein, we reflect upon the implications of the ongoing characterization of the SMYD family with regard to the growing landscape of therapeutic targets.
\end{abstract}

\section{Introduction}

In 1995, Hwang and Gottlieb reported the discovery of the SMYD1 gene as an unanticipated finding during their study of the CD8b promoter ${ }^{1}$. According to their report, unexpected promoter activity, distinct from that of $\mathrm{CD} 8 \mathrm{~b}$, was identified upstream of the $\mathrm{CD} 8 \mathrm{~b}$ gene. Further analysis ultimately revealed that the second promoter activity was associated with a gene that is transcribed opposite to that of CD8b. The newly detected gene was thus reported with the designation, "Bop," for "CD8b opposite." It was not for another decade, when a second member of the family was reported ${ }^{2}$, that the official nomenclature for the Bop family was revised to account for the unique presence of both a SET (Suppressor of variegation, Enhancer of Zeste, Trithorax $)^{3}$ and MYND (Myeloid-Nervy-DEAF1) ${ }^{4-6}$ domain. Since then, the SMYD family has been characterized as key regulators of cellular processes whose aberrant expression can lead to pathological consequences.

\section{SMYD1}

Following a targeted deletion of SMYD1, Gottlieb et al. ultimately determined that it is essential in cardiomyocyte differentiation and cardiac morphogenesis $^{7}$. Absence of SMYD1 reduces the expression of key transcription factors associated with the right ventricle of the heart, leading to ventricular hypoplasia. In a murine model, this is embryonically lethal at day 9.5. The transcriptional regulation imparted by SMYD1 was later shown to be facilitated by the methylation activity of its SET domain $^{8}$. This finding, along with the more recent elucidation of the SMYD1 structure $^{9}$, provide a foundation upon which future advances may lead to therapeutics for the clinical management or, perhaps, prevention of human heart defects involving the underexpression of SMYD1. Given the timing of events dependent upon proper expression of SMYD1 during embryogenesis, likely pathways for gene therapeutic intervention are dependent upon significant advances in gene therapy technologies.

\section{SMYD2}

The initial characterization of SMYD2 indicated that it too catalyzes methylation in a highly specific, SETdependent manner ${ }^{10}$. That study also revealed that SMYD2, like SMYD1, is highly expressed in the heart. However, targeted deletion of SMYD2 in a murine model showed that it is dispensable in the heart ${ }^{11}$. The greatest therapeutic potential associated with SMYD2 is likely related to its role in the SETmediated methylation and repression of the tumor suppressor, p53. More recently, the retinoblastoma tumor suppressor has also been identified as a target of SMYD2. Thus potential therapeutics involving SMYD2 will likely have oncogenic applications via spatial and/or temporal control SMYD2-mediated catalysis. Studies highlighting the structural basis for

This article is published under the terms of the Creative Commons Attribution License 4.0 Author(s) retain the copyright of this article. Publication rights with Alkhaer Publications. Published at: http://www.ijsciences.com/pub/issue/2015-12/

DOI: $10.18483 /$ ijSci.878; Online ISSN: 2305-3925; Print ISSN: 2410-4477 
SMYD2-mediated methylation of $\mathrm{p} 53^{12-13}$ provide a

Figure 1: Ribbon structure of SMYD2

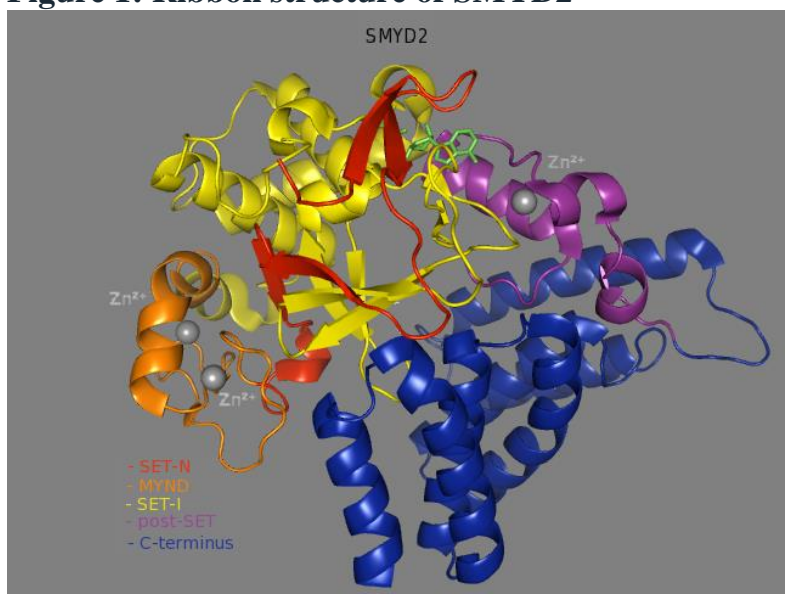

\section{SMYD3}

SMYD3 has been shown to catalyze trimethylation of $\mathrm{H} 4-\mathrm{K} 20^{14}$, $\mathrm{H} 4-\mathrm{K} 5^{15}$, and $\mathrm{H} 3-\mathrm{K} 4^{2}$ and monomethylation of vascular endothelial growth factor receptor $1^{16}$. SMYD3 has also been implicated as a proto-oncogene in colorectal, hepatocellular and breast carcinomas ${ }^{17-20}$ by virtue of its over-expression and promoter-associated polymorphisms in malignant cells. Thus, catalytic inhibition of aberrantly expressed SMYD3 is the target of most ongoing attempts to develop therapeutics involving this SMYD family member. Targeting of its catalytic domain has been facilitated by the availability of three independent SMYD3 crystal complexes ${ }^{14,21-22}$.

\section{Figure 2: Ribbon structure of SMYD3}

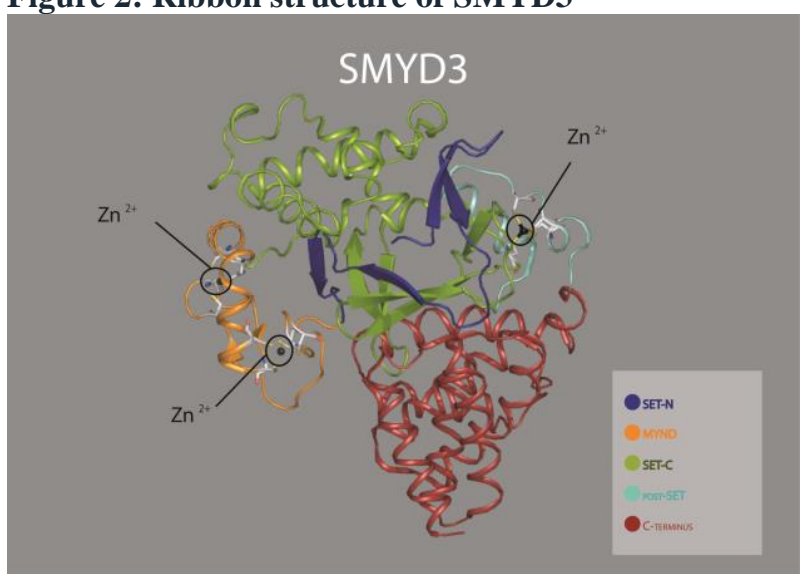

Further potential for therapeutic applications associated with SMYD3 are manifest in the recent findings that a degenerate tetratricopeptide repeat (TPR)-like domain encoded in the SMYD3 Cterminal domain (CTD) mediates physical interaction with the nuclear chaperone, HSP90 ${ }^{23}$. In the same study, it is further demonstrated that the CTD of SMYD3 is essential for its basal HMTase activity and that the TPR-like structure facilitates HSP90enhanced catalysis. Finally, impairment of the framework for such translational research.

SMYD3-HSP90 association results in SMYD3 mislocalization within the nucleus and its consequent loss of chromatin association. This, in turn, results in reduction of SMYD3-mediated cell proliferation and inhibition of its oncogenic activity. These findings highlight the potential for a novel approach to impeding HSP90-driven malignancy in SMYD3overexpressing cells which would exhibit a reduced toxicity profile over current HSP90 inhibitors.

\section{SMYD4 and SMYD5}

SMYD4 and SMYD5 have yet to be comprehensively characterized. However, data from Expressed Sequence Tags suggests that they are expressed in a wide range of normal, tumor, and diseased tissues ${ }^{24}$. This broad range of expression presents the potential for future applications targeting the expression or catalytic mechanisms of SMYD4 and/or SMYD5 in the clinical management of human disease.

\section{Conclusions}

The SMYD Family is a group of cell regulators that function primarily through SET-mediated methylation of target proteins. Future research on SMYD proteins, with strong emphasis on the distinct organismal context, will further elucidate key biological functions of SMYD family proteins along with the implications of their aberrant activities. Such emphases may reveal new paradigms for framing future therapeutics targeting the expression and/or catalytic activity of the SMYD family.

\section{Acknowledgments}

The authors gratefully acknowledge support from the National Science Foundation (1060548).

\section{References}

1. Hwang I and Gottlieb PD. Bop: a new T-cell-restricted gene located upstream of and opposite to mouse CD8b. Immunogenetics. 1995; 42(5): 353-61.

2. Hamamoto R, Furukawa Y, Morita M, Iimura Y, Silva FP, Li M, Yagyu R and Nakamura Y. SMYD3 encodes a histone methyltransferase involved in the proliferation of cancer cells, Nat Cell Biol, 2004; 6: 731-740.

3. Alvarez-Venegas R and Avramova Z. SET-domain proteins of the $\mathrm{Su}(\mathrm{var}) 3-9, \mathrm{E}(\mathrm{z})$ and Trithorax families. Gene. 2002; 285: 25-37.

4. Veraksa A, Kennison J, McGinnis W. DEAF-1 function is essential for the early embryonic development of Drosophila. Genesis. 2002; 33:67-76.

5. Spadaccini R, Perrin H, Bottomley MJ, Ansieau S, Sattler M. Structure and functional analysis of the MYND domain. J Mol Biol. 2006; 358:498-508.

6. Liu Y, Cheney MD, Gaudet JJ, Chruszcz M, Lukasik SM, Sugiyama D, Lary J, Cole J, Dauter Z, Minor W, Speck NA, Bushweller JH. The tetramer structure of the Nervy homology two domain, NHR2, is critical for AML1/ETO's activity. Cancer Cell. 2006; 9:249-60.

7. Gottlieb PD, Pierce SA, Sims RJ, Yamagishi H, Weihe EK, Harriss JV, Maika SD, Kuziel WA, King HL and Olson EN. 
Bop encodes a muscle-restricted protein containing MYND and SET domains and is essential for cardiac differentiation and morphogenesis. Nat. Genet. 2002; 31: 25-32.

8. Tan X, Rotllant J, Li H, de Deyne P and Du SJ. SmyD1, a histone methyltransferase, is required for myofibril organization and muscle contraction in zebrafish embryos. Proc. Natl. Acad. Sci. 2006; 103: 2713-2718.

9. $\quad$ Sirinupong N, Brunzelle J, Ye J, Pirzada A, Nico L and Yang Z. Crystal structure of cardiac specific histone methyltransferase SmyD1 reveals unusual active site architecture. J. Biol. Chem. 2010; 285: 40635-40644.

10. Brown MA, Sims RJ, Gottlieb PD and Tucker PW. Identification and characterization of Smyd2: A split SET/MYND domain-containing histone H3 lysine 36specific methyltransferase that interacts with the Sin 3 histone deacetylase complex. Mol. Cancer. 2006; 5: 26.

11. Diehl F, Brown MA, van Amerongen MJ, Novoyatleva T, Wietelmann A, Harriss J, Ferrazzi F, Böttger T, Harvey RP, Tucker PW and Engel FB. Cardiac deletion of Smyd2 is dispensable for mouse heart development. PLoS One. 2010; $5: 3$.

12. Wang L, Li L, Zhang H, Luo X, Dai J, Zhou S, Atadja P, Lu C, Li E and Zhao K. Structure of Human SMYD2 Protein Reveals the Basis of p53 Tumor Suppressor Methylation. J. Biol. Chem. 2011; 286:38725-37.

13. Jiang Y, Sirinupong N, Brunzelle J and Yang Z. Crystal Structures of Histone and p53 Methyltransferase SmyD2 Reveal a Conformational Flexibility of the Autoinhibitory CTerminal Domain. PLoS ONE. 2011; 6:e21640.

14. Foreman KW, Brown M, Park F, Emtage S, Harriss J, Das C, Zhu L, Crew A, Arnold L, Shaaban S and Tucker P. Structural and Functional Profiling of the Human Histone Methyltransferase SMYD3. PLoS ONE. 2011; 6:1-12.

15. Van Aller GS, Reynoird N, Barbash O, Huddleston M, Liu S, Zmoos AF, McDevitt P, Sinnamon R, Le B, Mas G, Annan R, Sage J, Garcia BA, et al. Smyd3 regulates cancer cell phenotypes and catalyzes histone $\mathrm{H} 4$ lysine 5 methylation. Epigenetics. 2012; 7:340-3.

16. Kunizaki M, Hamamoto R, Silva FP, Yamaguchi K, Nagayasu T, Shibuya M, Nakamura Y and Furukawa Y. The Lysine 831 of Vascular Endothelial Growth Factor Receptor 1 is a Novel Target of Methylation by SMYD3. Cancer Research. 2007; 67:10759-65.

17. Silva FP, Hamamoto R, Kunizaki M, Tsuge M, Nakamura Y and Furukawa Y. Enhanced methyltransferase activity of SMYD3 by the cleavage of its N-terminal region in human cancer cells. Oncogene. 2008; 27:2686-92.

18. Hamamoto R, Silva FP, Tsuge M, Nishidate T, Katagiri T, Nakamura Y and Furukawa Y. Enhanced SMYD3 expression is essential for the growth of breast cancer cells. Cancer Sci. 2006; 97:113-8.

19. Tsuge M, Hamamoto R, Silva FP, Ohnishi Y, Chayama K, Kamatani N, Furukawa Y and Nakamura Y. A variable number of tandem repeats polymorphism in an E2F-1 binding element in the $5^{\prime}$ flanking region of SMYD3 is a risk factor for human cancers. Nat Genet. 2005; 37:1104-7.

20. Wang H, Liu Y, Tan W, Zhang Y, Zhao N, Jiang Y, Lin C, Hao B, Zhao D, Qian J, Lu D, Jin L, Wei Q, et al. Association of the variable number of tandem repeats polymorphism in the promoter region of the SMYD3 gene with risk of esophageal squamous cell carcinoma in relation to tobacco smoking. Cancer Sci. 2008; 99:787-91.

21. Sirinupong N, Brunzelle J, Doko E and Yang Z. Structural Insights into the Autoinhibition and Posttranslational Activation of Histone Methyltransferase SmyD3. J Mol Biol. 2011; 406:149-59.

22. $\mathrm{Xu} \mathrm{S}, \mathrm{Wu}$ J, Sun B, Zhong $\mathrm{C}$ and Ding J. Structural and biochemical studies of human lysine methyltransferase Smyd3 reveal the important functional roles of its post-SET and TPR domains and the regulation of its activity by DNA binding. Nucleic Acids Research. 2011; 39:4438-49.

23. Brown MA, Foreman K, Harriss J, Das C, Zhu L, Edwards M, Shaaban S, and Tucker H. C-terminal domain of SMYD3 serves as a unique HSP90-regulated motif in oncogenesis.
Oncotarget, In Press.

24. Leinhart $\mathrm{K}$ and Brown MA. SET/MYND Lysine Methyltransferases Regulate Gene Transcription and Protein Activity. Genes. 2011; 2:210-8. 